\title{
Bounds on Normal Approximation on a Half Plane in Multidimension
}

\author{
D. Thongtha \\ Department of Mathematics, Faculty of Science, Chulalongkorn University, Bangkok 10330, Thailand \\ E-mail: dthongtha@gmail.com \\ K. Neammanee (Corresponding author) \\ Department of Mathematics, Faculty of Science, Chulalongkorn University, Bangkok 10330, Thailand \\ E-mail: kritsana.n@chula.ac.th
}

Received: October 8, 2011 Accepted: October 21, 2011 Published: February 1, 2012

doi:10.5539/jmr.v4n1p9 URL: http://dx.doi.org/10.5539/jmr.v4n1p9

The research is financed by the Office of the Higher Education Commission, Thailand.

Abstract

We give bounds on multidimensional Berry-Esseen theorem on a set $A_{k}(x)=\left\{\left(w_{1}, w_{2}, \ldots, w_{k}\right) \in \mathbb{R}^{k} \mid \sum_{i=1}^{k} w_{i} \leq x\right\}$ for $x \in \mathbb{R}$ by using the Berry-Esseen theorem in $\mathbb{R}$. The rates of convergence are $O\left(n^{-\frac{1}{2}}\right)$. In addition, we give known constants in the bounds of the approximation.

Keywords: Berry-Esseen inequality, Central limit theorem, Uniform and non-uniform bounds

\section{Introduction}

For $n \in \mathbb{N}$, let $X_{i}, 1 \leq i \leq n$ be independent and identically distributed random variables with zero means and $\sum_{i=1}^{n} E X_{i}^{2}=1$. Define

$$
S_{n}=\sum_{i=1}^{n} X_{i}
$$

and $\Phi_{1}$ the standard normal distribution in $\mathbb{R}$. Suppose that $E\left|X_{i}\right|^{3}<\infty$ for $1 \leq i \leq n$. The uniform and non-uniform versions of the Berry-Esseen inequality are

$$
\sup _{x \in \mathbb{R}}\left|P\left(S_{n} \leq x\right)-\Phi_{1}(x)\right| \leq C_{0} \sum_{i=1}^{n} E\left|X_{i}\right|^{3}
$$

and

$$
\left|P\left(S_{n} \leq x\right)-\Phi_{1}(x)\right| \leq \frac{C_{1}}{1+|x|^{3}} \sum_{i=1}^{n} E\left|X_{i}\right|^{3}
$$

respectively, where $C_{0}$ and $C_{1}$ are positive constants. The uniform version was independently discovered by (Berry, 1941, p. 122-136) and (Esseen, 1945, p. 1-125) and the non-uniform version was discovered by (Nagaev, 1965, p. 214-235). Without assuming the identically of $X_{i}$, the best constant $C_{0}$ and $C_{1}$ were given by (Shevtsova, 2010, p. 862-864) and (Paditz, 1989, p. 453-464), respectively. The results are as follows:

Theorem 1.1 (Shevtsova, 2010, p. 862-864) Let $X_{i}, 1 \leq i \leq n$, be independent random variables such that $E X_{i}=0$ and $E\left|X_{i}\right|^{3}<\infty$. Assume that $\sum_{i=1}^{n} E X_{i}^{2}=1$. Then

$$
\sup _{x \in \mathbb{R}}\left|P\left(S_{n} \leq x\right)-\Phi_{1}(x)\right| \leq 0.5600 \sum_{i=1}^{n} E\left|X_{i}\right|^{3} .
$$


Theorem 1.2 (Paditz, 1989, p. 453-464) Under the assumptions of theorem 1.1, we have

$$
\left|P\left(S_{n} \leq x\right)-\Phi_{1}(x)\right| \leq \frac{31.935}{1+|x|^{3}} \sum_{i=1}^{n} E\left|X_{i}\right|^{3}
$$

for all real numbers $x$.

(Chen, 2001, p. 236-254) relaxed the condition to the finiteness of the second moments and gave uniform and non-uniform versions of the inequality. The constant of the non-uniform version was given by (Neammanee, 2007, p. 1-10). Here are the results.

Theorem 1.3 (Chen, 2001, p. 236-254) Let $X_{i}, 1 \leq i \leq n$, be independent random variables such that $E X_{i}=0$ and $\sum_{i=1}^{n} E X_{i}^{2}=1$. Then

$$
\sup _{x \in \mathbb{R}}\left|P\left(S_{n} \leq x\right)-\Phi_{1}(x)\right| \leq 4.1 \sum_{i=1}^{n}\left\{E\left|X_{i}\right|^{2} I\left(\left|X_{i}\right|>1\right)+E\left|X_{i}\right|^{3} I\left(\left|X_{i}\right| \leq 1\right)\right\}
$$

and for all real numbers $x$,

$$
\left|P\left(S_{n} \leq x\right)-\Phi_{1}(x)\right| \leq C \sum_{i=1}^{n}\left\{\frac{E\left|X_{i}\right|^{2} I\left(\left|X_{i}\right|>1+|x|\right)}{1+|x|^{2}}+\frac{E\left|X_{i}\right|^{3} I\left(\left|X_{i}\right| \leq 1+|x|\right)}{1+|x|^{3}}\right\} .
$$

Theorem 1.4 (Neammanee, 2007, p. 1-10) Under the assumptions of theorem 1.3, we have

$$
\left|P\left(S_{n} \leq x\right)-\Phi_{1}(x)\right| \leq C \sum_{i=1}^{n}\left\{\frac{E\left|X_{i}\right|^{2} I\left(\left|X_{i}\right|>1+|x|\right)}{1+|x|^{2}}+\frac{E\left|X_{i}\right|^{3} I\left(\left|X_{i}\right| \leq 1+|x|\right)}{1+|x|^{3}}\right\}
$$

where

$$
C= \begin{cases}13.11 & \text { if } 0 \leq|x|<1.3 \\ 28.54 & \text { if } 1.3 \leq|x|<2 \\ 46.32 & \text { if } 2 \leq|x|<3 \\ 61.40 & \text { if } 3 \leq|x|<7.98 \\ 40.12 & \text { if } 7.98 \leq|x|<14 \\ 39.39 & \text { if }|x| \geq 14\end{cases}
$$

The reduction they make is truncation. This method make the random variables become bounded random varibles. In the case that each $X_{i}$ is bounded, the uniform and non-uniform versions were given in (Chen, 2005, p. 1-59) and (Chaidee, 2005), respectively.

Theorem 1.5 (Chen, 2005, p. 1-59) Let $X_{i}, 1 \leq i \leq n$, be independent random variables such that $E X_{i}=0, \sum_{i=1}^{n} E X_{i}^{2}=1$ and $\left|X_{i}\right| \leq \delta_{0}$, then

$$
\sup _{x \in \mathbb{R}}\left|P\left(S_{n} \leq x\right)-\Phi_{1}(x)\right| \leq 3.3 \delta_{0}
$$

Theorem 1.6 (Chaidee, 2005) Under the assumptions of theorem 1.5, there exists a constant $C$ which does not depend on $\delta_{0}$ such that for every real numbers $x$,

$$
\left|P\left(S_{n} \leq x\right)-\Phi_{1}(x)\right| \leq \frac{C \delta_{0}}{1+|x|^{3}} .
$$

For multidimensional case, let $k \in \mathbb{N}$ and $Y_{i}=\left(Y_{i 1}, Y_{i 2}, \ldots, Y_{i k}\right)$ be independent and identically distributed random vectors in $\mathbb{R}^{k}$ with zero means and covariance identity matrices $I_{k}$. Define

$$
W_{n}=\frac{1}{\sqrt{n}} \sum_{i=1}^{n} Y_{i}
$$


Let $F_{n}$ be the distribution function of $W_{n}$ and $\Phi_{k}$ the standard Gaussian distribution in $\mathbb{R}^{k}$. (Bergström, 1945, p. 106-127) guaranteed that $F_{n}$ converges weakly to $\Phi_{k}$ for large $n$. The uniform and non-uniform bounds of this convergence have been repeatedly refined over subsequent decades by many researchers such as (Esseen, 1945, p. 1-125), (Rao, 1961, p. 359-361), (Bahr, 1967, p. 61-69), (Bahr, 1967, p. 71-88) and (Bhattacharya, 1970, p. 68-86), etc. For the assumption that

$$
\sum_{j=1}^{k} E\left|Y_{1 j}\right|^{4}<\infty
$$

(Esseen, 1945, p. 1-125) gave a uniform bound on this convergence which is of the form

$$
\left|F_{n}\left(B_{r}\right)-\Phi_{k}\left(B_{r}\right)\right| \leq \frac{C}{n^{\frac{k}{k+1}}}
$$

where $B_{r}=\left\{\left(w_{1}, w_{2}, \ldots, w_{k}\right) \in \mathbb{R}^{k} \mid w_{1}^{2}+w_{2}^{2}+\cdots+w_{k}^{2} \leq r^{2}\right\}$ for $r>0$ and $C$ is an absolute constant depending only on the moment. (Rao, 1961, p. 359-361) generalized Esseen's result to any measurable convex subset $A$ of $\mathbb{R}^{k}$. His result is

$$
\left|F_{n}(A)-\Phi_{k}(A)\right| \leq \frac{C}{\sqrt{n}}(\log n)^{\frac{k-1}{2(k+1)}} .
$$

(Bahr, 1967, p. 71-88) assumed

$$
E\left(\sum_{j=1}^{k} Y_{1 j}^{2}\right)^{\frac{s}{2}}<\infty
$$

for an integer $s>k>1$ and improved the rate of convergence in (1) by the inequality

$$
\left|F_{n}(B)-\Phi_{k}(B)\right| \leq \frac{C}{\sqrt{n}} .
$$

In the case that each $Y_{i}$ may not be identically distributed random vectors, (Bhattacharya, 1970, p. 68-86) assumed

$$
\sum_{j=1}^{k} E\left|Y_{i j}\right|^{3+\delta}<\infty \text { for } 1 \leq i \leq n \text { where } \delta>0
$$

and gave a bound of the approximation as in (2) on any Borel subset of $\mathbb{R}^{k}$.

For a non-uniform version, (Bahr, 1967, p. 61-69) is the first one who investigated this version. He assumed the identically assumption on each $Y_{i}$ and gave the rate of convergence on $B_{k}(r)$. Under the finiteness assumption of the $s^{\text {th }}$ moments,

$$
E\left(\sum_{j=1}^{k} Y_{1 j}^{2}\right)^{\frac{s}{2}}<\infty
$$

for integer $s \geq 3$, the result is

$$
\left|F_{n}\left(B_{k}(r)\right)-\Phi_{k}\left(B_{k}(r)\right)\right| \leq \frac{C \cdot d(n)}{r^{s} n^{\frac{s-2}{2}}} \quad \text { for } \quad r \geq\left(\frac{5}{4} m(s-2) \log n\right)^{\frac{1}{2}}
$$

where $m$ is the largest eigenvalue of the covariance matrix of $Y_{i}, d(n)$ is bounded by one and $\lim _{n \rightarrow \infty} d(n)=0$. The aim of this paper is to find bounds on normal approximation to the distribution of $W_{n}$ over the set

$$
A_{k}(x)=\left\{\left(w_{1}, w_{2}, \ldots, w_{k}\right) \in \mathbb{R}^{k} \mid \sum_{i=1}^{k} w_{i} \leq x\right\}
$$

In this work, assume only that $\frac{1}{n k} \operatorname{Var}\left(\sum_{i=1}^{n} \sum_{j=1}^{k} Y_{i j}\right)=1$ and give our results on various assumptions, the random variables $Y_{i j}$ are bounded, $E\left|Y_{i j}\right|^{3}<\infty$ and $E\left|Y_{i j}\right|^{p}<\infty$ for some $2<p<3$. Our results are as follows: 
Theorem 1.7 If $\left|Y_{i j}\right| \leq \delta_{0}$ for $1 \leq i \leq n$ and $1 \leq j \leq k$, then

$$
\sup _{x \in \mathbb{R}}\left|P\left(W_{n} \in A_{k}(x)\right)-\Phi_{k}\left(A_{k}(x)\right)\right| \leq \frac{3.3 \sqrt{k} \delta_{0}}{\sqrt{n}}
$$

and there exists a constant $C$ which does not depend on $\delta_{0}$ such that for every real numbers $x$,

$$
\left|P\left(W_{n} \in A_{k}(x)\right)-\Phi_{k}\left(A_{k}(x)\right)\right| \leq \frac{C k^{2} \delta_{0}}{\sqrt{n}\left[(\sqrt{k})^{3}+|x|^{3}\right]} .
$$

Theorem 1.8 If $E\left|Y_{i j}\right|^{p}<\infty$ for $2<p<3,1 \leq i \leq n$ and $1 \leq j \leq k$, then

$$
\sup _{x \in \mathbb{R}}\left|P\left(W_{n} \in A_{k}(x)\right)-\Phi_{k}\left(A_{k}(x)\right)\right| \leq \frac{75(4)^{p-1} k^{p}}{(n k)^{\frac{p}{2}}} \sum_{i=1}^{n} \sum_{j=1}^{k} E\left|Y_{i j}\right|^{p}
$$

and there exists an absulute constant $C$ such that for $x \in \mathbb{R}$,

$$
\left|P\left(W_{n} \in A_{k}(x)\right)-\Phi_{k}\left(A_{k}(x)\right)\right| \leq \frac{5^{p} C k^{p}}{n^{\frac{p}{2}}(\sqrt{k}+|x|)^{p}} \sum_{i=1}^{n} \sum_{j=1}^{k} E\left|Y_{i j}\right|^{p} .
$$

Theorem 1.9 If $E\left|Y_{i j}\right|^{3}<\infty$ for $1 \leq i \leq n$ and $1 \leq j \leq k$, then

$$
\sup _{x \in \mathbb{R}}\left|P\left(W_{n} \in A_{k}(x)\right)-\Phi_{k}\left(A_{k}(x)\right)\right| \leq \frac{0.5600 \sqrt{k}}{n^{\frac{3}{2}}} \sum_{i=1}^{n} \sum_{j=1}^{k} E\left|Y_{i j}\right|^{3}
$$

and for all real numbers $x$

$$
\left|P\left(W_{n} \in A_{k}(x)\right)-\Phi_{k}\left(A_{k}(x)\right)\right| \leq \frac{31.935 k^{2}}{n^{\frac{3}{2}}\left[(\sqrt{k})^{3}+|x|^{3}\right]} \sum_{i=1}^{n} \sum_{j=1}^{k} E\left|Y_{i j}\right|^{3} .
$$

The proofs of our main theorems are given in the next section.

\section{Proof of Main Theorems}

In the proofs of main theorems, we use the Berry-Esseen theorems in $\mathbb{R}$ in which the limit distribution is $\Phi_{1}$. However, the limit distribution in our theorems is the standard Gaussian distribution $\Phi_{k}$ in $\mathbb{R}^{k}$. In the following proposition, we give a relation between $\Phi_{1}$ and $\Phi_{k}$.

Proposition 2.1 For $k \in \mathbb{N}$ and $x \in \mathbb{R}$, we have

$$
\Phi_{k}\left(A_{k}(x)\right)=\Phi_{1}\left(\frac{x}{\sqrt{k}}\right) .
$$

Proof: To prove the proposition, we let $B=\left\{b_{1}, b_{2}, \ldots, b_{k}\right\}$ be an orthonorrmal basis for $\mathbb{R}^{k}$ with $b_{1}=\frac{1}{\sqrt{k}}(1,1, \ldots, 1)$ and $w=\left(w_{1}, w_{2}, \ldots, w_{k}\right) \in A_{k}(x)$. Set

$$
t_{1}=\left\langle b_{1}, w\right\rangle \text { and } t_{i}=\left\langle b_{i}, w\right\rangle \text { for } t=2,3, \ldots, k
$$

Then

$$
\begin{gathered}
t_{1}=\frac{1}{\sqrt{k}} \sum_{i=1}^{k} w_{i} \leq \frac{x}{\sqrt{k}},-\infty<t_{i}<\infty, \text { for } t=2,3, \ldots, k, \text { and } \\
\sum_{i=1}^{k}\left\langle b_{i}, w\right\rangle b_{i}=w=\sum_{i=1}^{k}\left\langle e_{i}, w\right\rangle e_{i}
\end{gathered}
$$

where $\left\{e_{1}, e_{2}, \ldots, e_{k}\right\}$ is a usual orthonormal basis for $\mathbb{R}^{k}$. Thus, we have

$$
\begin{aligned}
\sum_{i=1}^{k} w_{i}^{2} & =\left\|\sum_{i=1}^{k} w_{i} e_{i}\right\|^{2}=\left\|\sum_{i=1}^{k}\left\langle e_{i}, w\right\rangle e_{i}\right\|^{2}=\left\|\sum_{i=1}^{k}\left\langle b_{i}, w\right\rangle b_{i}\right\|^{2}=\left\|\sum_{i=1}^{k} t_{i} b_{i}\right\|^{2} \\
& =\sum_{i=1}^{k} t_{i}^{2} .
\end{aligned}
$$


Let $J$ be the Jacobian matrix,

$$
J=\left[\begin{array}{cccc}
\frac{\partial w_{1}}{\partial t_{1}} & \frac{\partial w_{2}}{\partial t_{1}} & \cdots & \frac{\partial w_{k}}{\partial t_{1}} \\
\frac{\partial w_{1}}{\partial t_{2}} & \frac{\partial w_{2}}{\partial t_{2}} & \cdots & \frac{\partial w_{k}}{\partial t_{2}} \\
\vdots & \vdots & \cdots & \vdots \\
\frac{\partial w_{1}}{\partial t_{k}} & \frac{\partial w_{2}}{\partial t_{k}} & \cdots & \frac{\partial w_{k}}{\partial t_{k}}
\end{array}\right] .
$$

Thun $|\operatorname{det}(J)|=1$. Then by (3), we have

$$
\begin{aligned}
\Phi_{k}\left(A_{k}(x)\right) & =\frac{1}{(2 \pi)^{\frac{k}{2}}} \iint \cdots \int_{A_{k}(x)} e^{-\frac{1}{2} \sum_{i=1}^{k} w_{i}^{2}} d w_{1} d w_{2} \cdots d w_{k} \\
& =\frac{1}{(2 \pi)^{\frac{k}{2}}} \int_{-\infty}^{\infty} \int_{-\infty}^{\infty} \cdots \int_{-\infty}^{\infty} \int_{-\infty}^{\frac{x}{\sqrt{k}}} e^{-\frac{1}{2} \sum_{i=1}^{k} t_{i}^{2}}|\operatorname{det} J| d t_{1} d t_{2} \cdots d t_{k} \\
& =\frac{1}{\sqrt{2 \pi}} \int_{-\infty}^{\frac{x}{\sqrt{k}}} e^{-t^{2}} d t \\
& =\Phi_{1}\left(\frac{x}{\sqrt{k}}\right) .
\end{aligned}
$$

Hence, the proposition is proved.

The proof of theorem 1.7 is completed by applying theorem 1.5-1.6. The proof of theorem 1.8, we use the propostion 2.1 and theorems in (Chen, 2004, p. 1985-2028). Theorem 1.9 is proved by applying theorem 1.1-1.2, respectively.

\section{Proof of theorem 1.7}

Proof: For each $1 \leq i \leq n$ and, $1 \leq j \leq k$, we define

$$
W_{j n}=\frac{1}{\sqrt{n}} \sum_{i=1}^{n} Y_{i j} \quad \text { and } \quad T_{i n}=\frac{1}{\sqrt{n}} \sum_{j=1}^{k} Y_{i j} .
$$

Thus $T_{1 n}, T_{2 n}, \ldots, T_{n n}$ are independent,

$$
\begin{gathered}
E\left(T_{i n}\right)=0, \quad\left|T_{i n}\right| \leq \frac{k \delta_{0}}{\sqrt{n}}, \\
W_{n}=\left(W_{1 n}, W_{2 n}, \ldots, W_{k n}\right) \quad \text { and } \quad \sum_{j=1}^{k} W_{j n}=\sum_{i=1}^{n} T_{i n} .
\end{gathered}
$$

Since $Y_{i}$ has zero mean and covariance matrix $I_{k}$,

$$
\operatorname{Var}\left(Y_{i j}\right)=1 \quad \text { and } \operatorname{Cov}\left(Y_{i j}, Y_{i k}\right)=0 \quad \text { for } \quad j \neq k .
$$

Therefore

$$
\operatorname{Var}\left(\frac{1}{\sqrt{k}} \sum_{i=1}^{n} T_{i n}\right)=\frac{1}{k} \operatorname{Var} \sum_{i=1}^{n} T_{i n}=\frac{1}{n k} \operatorname{Var} \sum_{i=1}^{n} \sum_{j=1}^{k} Y_{i j}=1 .
$$

By applying theorem 1.5 , proposition 2.1 and (4)-(6), we have

$$
\begin{aligned}
\sup _{x \in \mathbb{R}}\left|P\left(W_{n} \in A_{k}(x)\right)-\Phi_{k}\left(A_{k}(x)\right)\right| & =\sup _{x \in \mathbb{R}}\left|P\left(\sum_{j=1}^{k} W_{j n} \leq x\right)-\Phi_{1}\left(\frac{x}{\sqrt{k}}\right)\right| \\
& =\sup _{x \in \mathbb{R}}\left|P\left(\sum_{i=1}^{n} T_{i n} \leq x\right)-\Phi_{1}\left(\frac{x}{\sqrt{k}}\right)\right| \\
& =\sup _{x \in \mathbb{R}}\left|P\left(\frac{1}{\sqrt{k}} \sum_{i=1}^{n} T_{i n} \leq \frac{x}{\sqrt{k}}\right)-\Phi_{1}\left(\frac{x}{\sqrt{k}}\right)\right| \\
& \leq \frac{3.3 \sqrt{k} \delta_{0}}{\sqrt{n}} .
\end{aligned}
$$


For the second part, we apply theorem 1.6. The result is

$$
\begin{aligned}
\left|P\left(W_{n} \in A_{k}(x)\right)-\Phi_{k}\left(A_{k}(x)\right)\right| & =\left|P\left(\frac{1}{\sqrt{k}} \sum_{i=1}^{n} T_{i n} \leq \frac{x}{\sqrt{k}}\right)-\Phi_{1}\left(\frac{x}{\sqrt{k}}\right)\right| \\
& \leq \frac{C \sqrt{k} \delta_{0}}{\sqrt{n}\left(1+\left|\frac{x}{\sqrt{k}}\right|^{3}\right)} \\
& =\frac{C k^{2} \delta_{0}}{\sqrt{n}\left[(\sqrt{k})^{3}+|x|^{3}\right]} .
\end{aligned}
$$

for all real numbers $x$.

In theorem 1.8, we give the bounds by applying theorem 2.4 and theorem 2.5 in (Chen, 2004, p. 1985-2028). To prove this, we need the proposition 2.2. This proposition gives us that the random field $\left\{Y_{i, j} \mid i=1,2, \ldots, n, j=1,2, \ldots, k\right\}$ satisfied $\left(L D 4^{*}\right)$ in (Chen, 2004, p. 1985-2028). This condition is proposed as follows:

Let $\mathcal{J}$ be a finite index set of cardianality $n$, and let $\left\{X_{i}, i \in \mathcal{J}\right\}$ be a random field with zero means and finite variances. For $A \subset \mathcal{J}$, let $X_{A}$ denote $\left\{X_{i}, i \in A\right\}, A^{c}=\{j \in \mathcal{J}: j \notin A\}$ and $|A|$ the cardinality of $A$. The random field $\left\{X_{i}, i \in \mathcal{J}\right\}$ satisfied $\left(L D 4^{*}\right)$ if for each $i \in \mathcal{J}$ there exists $A_{i} \subset B_{i} \subset B_{i}^{*} \subset C_{i}^{*} \subset D_{i}^{*} \subset \mathcal{J}$ such that $X_{i}$ is independent of $X_{A_{i}^{c}}, X_{A_{i}}$ is independent of $X_{B_{i}^{c}}$ and then $X_{A_{i}}$ is independent of $\left\{X_{A_{j}}, j \in B_{i}^{* c}\right\},\left\{X_{A_{l}}, l \in B_{i}^{*}\right\}$ is independent of $\left\{X_{A_{j}}, j \in C_{i}^{* c}\right\}$ and $\left\{X_{A_{l}}, l \in C_{i}^{*}\right\}$ is independent of $\left\{X_{A_{j}}, j \in D_{i}^{* c}\right\}$.

Proposition 2.2 For $k, n \in \mathbb{N}$, let $Y_{i}=\left(Y_{i 1}, Y_{i 2}, \ldots, Y_{i k}\right), i=1,2, \ldots, n$ be independent random vectors in $\mathbb{R}^{k}$ with zero means. Then $\left\{Y_{i j} \mid i=1,2, \ldots, n, j=1,2, \ldots, k\right\}$ satisfies $\left(L D 4^{*}\right)$.

Proof: This proposition is completed by setting $A_{i j} \subset B_{i j} \subset B_{i j}^{*} \subset C_{i j}^{*} \subset D_{i j}^{*}$ for $i=1,2, \ldots, n$ and $j=1,2, \ldots, k$ as follows:

$$
\begin{gathered}
A_{i j}=\{i l \mid l=1,2, \ldots, k\} \text { for } i=1,2, \ldots, n, \\
B_{i j}=\{i l,(i+1) l \mid l=1,2, \ldots, k\} \text { for } i=1,2, \ldots, n-1 \text { and } B_{n j}=B_{(n-1) j}, \\
B_{i j}^{*}=C_{i j}=\{i l,,(i+1) l,(i+2) l \mid l=1,2, \ldots, k\} \text { for } i=1,2, \ldots, n-2 \text { and } \\
B_{(n-m) j}^{*}=C_{(n-m) j}=B_{(n-2) j} \text { for } m=1,2, \\
C_{i j}^{*}=\{i l,(i+1) l, \ldots,(i+3) l \mid l=1,2, \ldots, k\} \text { for } i=1,2, \ldots, n-3 \text { and } \\
C_{(n-m) j}^{*}=C_{(n-3) j}^{*} m=1,2,3, \\
D_{i j}^{*}=\{i l,(i+1) l, \ldots,(i+4) l \mid l=1,2, \ldots, k\} \text { for } i=1,2, \ldots, n-4 \text { and } D_{(n-m) j}^{*}=D_{(n-4) j}^{*} m=1,2,3,4 .
\end{gathered}
$$

So, we have the proposition.

From the sets defined in the above proposition, we can compute directly that for each $i=1,2, \ldots, n$,

$$
\max \left(\left|N\left(C_{i}\right)\right|,\left|\left\{j: i \in C_{j}\right\}\right| \leq 4\right.
$$

and

$$
\max _{1 \leq i \leq n} \max \left(\left|D_{i}^{*}\right|,\left|\left\{j: i \in D_{j}^{*}\right\}\right|\right) \leq 5
$$

where $N\left(C_{i}\right)$ is defined in theorem 2.3 in (Chen, 2004, p. 1985-2028).

The condition $\left(L D 4^{*}\right)$ implies the condition $(L D 3)$ in (Chen, 2004, p. 1985-2028). Thus $\left\{Y_{i j} \mid i=1,2, \ldots, n, j=\right.$ $1,2, \ldots, k\}$ satisfies $(L D 3)$.

\section{Proof of theorem 1.8}

Proof: For each $1 \leq i \leq n$, define $T_{\text {in }}$ as in the proof of theorem 1.7. By the inequality

$$
\left|\sum_{j=1}^{k} Y_{i j}\right|^{p} \leq k^{p} \sum_{j=1}^{k}\left|Y_{i j}\right|^{p}
$$

we have

$$
E\left|T_{i n}\right|^{p}=\frac{1}{n^{\frac{p}{2}}} E\left|\sum_{j=1}^{k} Y_{i j}\right|^{p} \leq \frac{k^{p}}{n^{\frac{p}{2}}} \sum_{j=1}^{k} E\left|Y_{i j}\right|^{p}<\infty .
$$


So, by (4), (6), (8), (10), proposition 2.2 and theorem 2.4 in (Chen, 2004, p. 1985-2028), we have

$$
\begin{aligned}
\sup _{x \in \mathbb{R}}\left|P\left(W_{n} \in A_{k}(x)\right)-\Phi_{k}\left(A_{k}(x)\right)\right| & =\sup _{x \in \mathbb{R}}\left|P\left(\frac{1}{\sqrt{k}} \sum_{i=1}^{n} T_{i n} \leq \frac{x}{\sqrt{k}}\right)-\Phi_{1}\left(\frac{x}{\sqrt{k}}\right)\right| \\
& \leq 75(4)^{p-1} \sum_{i=1}^{n} E\left|\frac{T_{i}}{\sqrt{k}}\right|^{p} \\
& =\frac{75(4)^{p-1}}{(n k)^{\frac{p}{2}}} \sum_{i=1}^{n} E\left|\sum_{j=1}^{k} Y_{i j}\right|^{p} \\
& \leq \frac{75(4)^{p-1} k^{p}}{(n k)^{\frac{p}{2}}} \sum_{i=1}^{n} \sum_{j=1}^{k} E\left|Y_{i j}\right|^{p} .
\end{aligned}
$$

Applying theorem 2.5 in (Chen, 2004, p. 1985-2028) and (9) to non-uniform case, we have for $x \in \mathbb{R}$,

$$
\begin{aligned}
\left|P\left(W_{n} \in A_{k}(x)\right)-\Phi_{k}\left(A_{k}(x)\right)\right| & =\left|P\left(\sum_{i=1}^{n} T_{i n} \leq x\right)-\Phi_{1}\left(\frac{x}{\sqrt{k}}\right)\right| \\
& \leq \frac{5^{p} C}{\left(1+\left|\frac{x}{\sqrt{k}}\right|\right)^{p}} \sum_{i=1}^{n} E\left|\frac{T_{i}}{\sqrt{k}^{p}}\right|^{p} \\
& \leq \frac{(5 k)^{p} C}{n^{\frac{p}{2}}(\sqrt{k}+|x|)^{p}} \sum_{i=1}^{n} \sum_{j=1}^{k} E\left|Y_{i j}\right|^{p} .
\end{aligned}
$$

\section{Proof of theorem 1.9}

Proof: By theorem 1.1, 1.2 and the same argument as in theorem 1.7, we have the theorem.

Remark The above theorems include the case that each $Y_{i}$ has an indicator covariance matrix $I_{k}$.

\section{Acknowledgment}

The authors would like to thank the reviewers for their valuable comments and suggestions.

\section{References}

Bahr, B. V. (1967). Multi-dimentional integral limit theorems. Ark Mat., 7, 71-88. http://dx.doi.org/10.1007/BF02591678

Bahr, B. V. (1967). On the central limit theorem in $R_{k}$. Ark Mat., 7, 61-69. http://dx.doi.org/10.1007/BF02591677

Bergström, H. (1945). On the central limit theorem in $\mathbb{R}^{k}, k>1$. Skand. Akt., 28, 106-127.

Berry, A. C. (1941). The accuracy of the Gaussian approximation to the sum of independent variables. Trans. Amer. Math. Soc., 49, 122-136. http://dx.doi.org/10.1090/S0002-9947-1941-0003498-3

Bhattacharya, R. N. (1970). Rates of weak convergence for the multidimensional central limit theorem. Theory Probab. Appl., 15, 68-86. http://dx.doi.org/10.1137/1115005

Chaidee, N. (2004). Non-uniform Bounds in Normal Approximation for Matrix Correlation Statistics and Independent Bounded Random Variables, Ph. D. thesis, Chulalongkorn University

Chen, L. H. Y., \& Shao, Q. M. (2001). A non-uniform Berry-Esseen bound via Stein's method. Probab. Theory Relat. Field, 120, 236-254. http://dx.doi.org/10.1007/PL00008782

Chen, L. H. Y., \& Shao, Q. M. (2004). Normal approximation under local dependence. Ann. Probab., 32, 1985-2028. http://dx.doi.org/10.1214/009117904000000450

Chen, L. H. Y., \& Shao, Q. M. (2005). Stein's method for normal approximation. An Introduction to Stein's Method, 4 , 1-59, Singapore University Press, Singapore.

Esseen, C. G. (1945). Fourier analysis of distribution functions. A Mathematical Study of the Laplace Gaussian Law, Acta Math., 77, 1-125. http://dx.doi.org/10.1007/BF02392223

Nagaev, S. V. (1965). Some limit theorems for large deviations. Theory Probab. Appl., 10, 214-235. http://dx.doi.org/10.1137/1110027 
Neammanee K., \& Thongtha, P. (2007). Improvement of the non-uniform version of Berry-Esseen inequality via PaditzSiganov theorems. JIPAM, 8(4), 1-10.

Paditz, L. (1989). On the analytical structure of the constant in the nonuniform version of the Esseen inequality. Statistics, 20, 453-464. http://dx.doi.org/10.1080/02331888908802196

Rao, R. R. (1961). On the central limit theorem in $R_{k}$. Bull. Amer. Math. Soc., 67, 359-361. http://dx.doi.org/10.1090/S0002-9904-1961-10615-0

Shevtsova, I. G. (2010). An Improvement of convergence rate estimates in the Lyapunov Theorem. Dokl. Math., 82(3), 862-864. http://dx.doi.org/10.1134/S1064562410060062 